\title{
Tratamento Videolaparoscópico de Endometriomas Ovarianos
}

\author{
Videolaparoscopic Management of Ovarian Endometriomas
}

Adriana C.S. Lopez, Luciano L.R. Santos, José Francisco Dória Ramos, Salete Yatabe, Reginaldo Guedes C. Lopes, Umberto Gazi Lippi

\section{RESUM0}

\begin{abstract}
Objetivo: avaliar os resultados do tratamento videolaparoscópico de 32 pacientes com endometrioma ovariano.

Métodos: estudo retrospectivo que incluiu trinta e duas pacientes encaminhadas ao setor de Endoscopia Ginecológica do Serviço de Ginecologia e Obstetrícia do Hospital do Servidor Público Estadual "Francisco Morato de Oliveira" - São Paulo, as quais tinham diagnóstico clínico e ultra-sonográfico de endometrioma ovariano e foram tratadas por videolaparoscopia. Onze pacientes com endometriomas com diâmetro menor que $3 \mathrm{~cm}$ foram submetidas à drenagem do endometrioma e exérese da cápsula na primeira laparoscopia. Vinte e uma pacientes com endometriomas com diâmetros maior que $3 \mathrm{~cm}$ foram submetidas à drenagem do endometrioma e lavagem do cisto na primeira laparoscopia. A seguir, utilizaram análogo do GnRH por quatro meses (1 ampola mensal) e então foram submetidas à segunda laparoscopia para retirada da cápsula do endometrioma. O exame histopatológico do material obtido na cirurgia foi realizado em todos os casos. Avaliou-se o resultado imediato do procedimento e a freqüencia de recidivas. Resultados: não se registraram intercorrências cirúrgicas ou complicações pós-operatórias. Ocorreram três recidivas em um periodo de seis a doze meses, todas no grupo de 21 pacientes com endometriomas com o diâmetro maior que $3 \mathrm{~cm}$.

Conclusão: o tratamento videolaparoscópico dos endometriomas ovarianos com retirada da cápsula apresenta bons resultados e baixo indice de recidiva.
\end{abstract}

PALAVRAS-CHAVE: Endometriose. Laparoscopia. Análogos do GnRH.

Introdução

A endometriose é uma doença benigna do aparelho genital feminino caracterizada pela presença de tecido endometrial fora da cavidade uterina, que pode se apresentar de forma difusa, com implantes pélvicos ou de forma localizada (endometrioma). O endometrioma ovariano foi descrito pela primeira vez por Russel em 1899. Este autor observou em uma paciente menopausada aderência envolvendo o ovário direito e a face posterior do ligamento largo, cuja microscopia mostrou "áreas com estruturas exatamente iguais à mucosa uterina normal” .

Serviço de Ginecologia e Obstetrícia do Hospital do Servidor Público Estadual "Francisco Morato de Oliveira" - São Paulo SP

Correspondência:

Umberto Gazi Lippi

Rua Borges Lagoa, 564, conj 32 - Vila Clementino

04038-000 - São Paulo - SP
A etiologia do endometrioma ovariano é controversa. Admite-se tratar-se de um pseudocisto formado pela deposição de sangue menstrual na superfície do órgão. A invaginação deste material seria dependente de seu envolvimento por aderências. Brosens et al. ${ }^{2}$ sugeriram que o endometrioma verdadeiro seria causado por um foco de endometriose na periferia do ovário e posterior invaginação. Não haveria portanto uma cápsula e sim tecido da cortical ovariana, invaginado. $\mathrm{O}$ falso endometrioma seria resultado da presença de um foco de endometriose em um cisto de corpo lúteo. Haveria a penetração deste foco e formação de um cisto hemorrágico. Coerente com esta teoria, Brosens et al. ${ }^{3}$ propôs a destruição apenas do foco endometriótico na cortical invaginada, pois não haveria cápsula a ser retirada.

Nezhat et al. ${ }^{4}$ sugerem que os endometriomas teriam sua origem no envolvimento secun- 
dário do processo endometriótico em cistos funcionais. Donnez et al. ${ }^{5}$ adotam a teoria da metaplasia celômica em regiões de invaginação do epitélio celômico ovariano. Segundo estes autores, haveria uma metaplasia de células ovarianas em células endometriais.

O diagnóstico do endometrioma ovariano pode ser sugerido pela queixa clínica da paciente (dismenorréia, dor pélvica crônica, infertilidade) e pela ultra-sonografia. A confirmação se dá pela inspeção laparoscópica, ovarioscopia e exame histológico. Brosens et $\mathrm{al}^{2}{ }^{2}$ salientam o importante papel da ovarioscopia na obtenção de material para exame anatomopatológico.

As opções de tratamento incluem a conduta expectante, terapêutica medicamentosa ou cirúrgica a depender da clínica e da gravidade da doença. A maioria dos autores adota uma abordagem cirúrgica sobretudo para os casos de endometriomas com diâmetro maior que $3 \mathrm{~cm}$, devido à má resposta e aos altos índices de recidiva apenas com o bloqueio da função ovariana ${ }^{4,6}$. Deve-se, porém, ressaltar que as drogas bloqueadoras da função ovariana têm um papel importante quando utilizadas no pré-operatório por reduzirem a vascularização pélvica, o tamanho do cisto e a resposta inflamatória ${ }^{6}$, embora Muzii $^{7}$ não tenha demonstrado tais benefícios.

$\mathrm{Na}$ literatura descrevem-se várias técnicas para a abordagem cirúrgica do endometrioma ovariano. Donnez et al. ${ }^{5}$ e Sutton et al. ${ }^{8}$ propõem a vaporização da cápsula com laser de $\mathrm{CO}_{2}$. Muzii ${ }^{7}$ sugere a exérese da cápsula por via laparoscópica. Brosens et al. ${ }^{2}$ defendem a eversão da cápsula do endometrioma e a destruição apenas do foco endometriótico. Consideram lesiva ao ovário a tentativa de retirada da mesma. Busacca et al. ${ }^{9}$, com emprego da cistectomia, referem recidiva de 11,7\% em 366 pacientes. Saleh e Tulandi ${ }^{10}$, comparando a cistectomia com a drenagem simples do endometrioma, observaram recidiva de $6,1 \%$ no $1^{\circ}$ grupo e $21,9 \%$ no segundo, após 18 meses. Hemmings et al. ${ }^{11}$ afirmam não haver diferença quanto ao sucesso da cirurgia, taxas de gravidez e índice de recidiva praticando a retirada da cápsula ou cauterizando-a com corrente elétrica bipolar. Beretta et al. ${ }^{12}$ têm melhores resultados com a cistectomia do que com a cauterização da cápsula.

Neste trabalho são avaliados os resultados obtidos com o protocolo de abordagem do endometrioma ovariano utilizado no Serviço, conforme descrito adiante, o qual visa a preservação funcional lesando minimamente a gônada afetada.

\section{Pacientes e Métodos}

Foram analisados retrospectivamente os prontuários médicos de 32 pacientes encaminhadas ao setor de Endoscopia Ginecológica, entre 1993 e 1997, com queixa clínica de dor pélvica crônica e diagnóstico clínico e ultra-sonográfico de endometrioma, ovariano e com confirmação histopatológica posterior. A idade das mesmas variou de 23 a 43 anos.

A escolha do tipo de tratamento cirúrgico laparoscópico foi baseada no tamanho do endometrioma de acordo com o protocolo do Serviço. Foi usado um conjunto de laparoscopia da marca Stortz, com ótica de $10 \mathrm{~mm}$ de diâmetro. A retirada de cápsula foi feita com duas pinças prensoras denteadas de $5 \mathrm{~mm}$. Em 11 casos com endometriomas com diâmetro menor que $3 \mathrm{~cm}$ a cirurgia foi realizada em apenas um tempo com abertura, drenagem e retirada da cápsula. Nos 21 restantes com tumores com diâmetro maior que $3 \mathrm{~cm}$ o tratamento foi realizado em 2 tempos. Na primeira laparoscopia foi realizada apenas abertura e drenagem do endometrioma. Durante os 4 meses seguintes as pacientes foram tratadas com análogo do GnRH. Após este período, foram submetidas a nova laparoscopia para a retirada da cápsula. Não houve problema quanto à aceitação desta segunda intervenção, visto que as pacientes eram informadas previamente sobre essa possibilidade. Em todos os casos foi realizado exame histopatológico do material colhido durante o ato operatório. Analisaram-se os resultados imediatos da cirurgia, em ambos os grupos, e a freqüência de recidivas durante o acompanhamento que se estendeu até 24 meses.

\section{Resultados}

As cirurgias laparoscópicas transcorreram sem complicações e a retirada da cápsula do endometrioma foi possivel em todos os casos. As 21 pacientes que fizeram o tratamento com análogos do GnRH por quatro meses após a primeira cirurgia referiram algum sintoma decorrente do hipoestrogenismo, como fogachos, irritabilidade e insônia, mas não interromperam o tratamento nem necessitaram do uso de estrógeno.

Não ocorreram recidivas nas 11 pacientes com endometriomas de até três centímetros. Nas 21 com endometrioma com diâmetro maior que três centímetros foram registradas três recidivas. 
Duas foram detectadas nos primeiros seis meses após a segunda laparoscopia e uma após um ano. Estas pacientes foram reoperadas e a cápsula do endometrioma foi retirada novamente. Após um ano não haviam apresentado nova recidiva.

O exame histopatológico realizado em todos os casos fez a confirmação do diagnóstico.

\section{Discussão}

O endometrioma de ovário pode ser abordado por diversas técnicas cirúrgicas, de complexidade variável, geralmente com bons resultados ${ }^{3,4,7}$. A simples drenagem no entanto mostrou-se ineficaz na abordagem do endometrioma ovariano $^{1}$ e a maioria dos autores preconiza a retirada da cápsula como o melhor tratamento ${ }^{13}$. A destruição da cápsula com eletrocautério ou laser faz com que se prejudique o estudo anatomopatológico da totalidade da cápsula, pois embora se faça a biópsia, trata-se apenas de um fragmento. Por outro lado, o argumento de Donne $z^{1}$ de que a retirada da cápsula pode lesar o parênquima ovariano diminuindo a população de folículos tem uma lógica aparente, embora não haja comprovação desta teoria. No Serviço de Ginecologia e Obstetrícia do Hospital do Servidor Público Estadual de São Paulo prefere-se o protocolo já descrito no qual se procura ser cuidadoso com o ovário ao mesmo tempo em que se resseca a totalidade da cápsula. A necessidade de se realizar um segundo procedimento laparoscópico é uma desvantagem deste protocolo.

Os casos de endometrioma ovariano diagnosticados pela clínica e pela ultra-sonografia devem ter sua confirmação pela histopatologia. $\mathrm{Na}$ casuística de Nezhat et al. ${ }^{4}$, de uma série de 216 presumiveis endometriomas, 46 (21\%) eram cistos de corpo lúteo, 2 (1\%) teratomas císticos hemorrágicos e 9 (4\%) cistos serosos. Em 159 (73\%) houve confirmação histopatológica.

Quanto à recidiva dos endometriomas, de 32 casos da casuística de Nezhat et al. ${ }^{4}$ tratados cirurgicamente, houve recidiva em três (9\%), todas entre os casos com endometrioma com diâmetro maior que $3 \mathrm{~cm}$. Canis et al. ${ }^{13}$ em 1992 relatou $7 \%$ de recidiva, também em endometriomas com estas dimensões. Fayez e Vogel ${ }^{14}$ relatam no entanto recidiva de $22 \%$.

A explicação para a recidiva dos endometriomas seria, segundo Donnez ${ }^{1}$, a presença de focos de endometriose extracísticos profundos não-visualizados quando da cirurgia. Por outro lado, os análogos do GnRH parecem não agir tão bem no ovário ${ }^{6}$ porque a droga não consegue atingir o foco de endometriose pela fibrose que se estabelece, ou porque as células endometrióticas parecem ter sua programação genética própria. Outra explicação seria o fato de as células endometrióticas no ovário terem menos receptores hormonais e os receptores, mesmo que presentes, parecem ser biologicamente inativos. A utilização do análogo do GnRH tem como objetivo a redução do tamanho do cisto, facilitando o procedimento cirúrgico e determinando menor trauma ovariano ${ }^{6}$.

Como já foi citado, a literatura mostra a ocorrência de supressão incompleta das células endometrióticas no endometrioma ovariano, o que explicaria a recorrência após o término da terapia de supressão. Neste trabalho foi feita a associação do análogo no pré-operatório de endometriomas grandes, com a posterior retirada cirúrgica da cápsula. A intenção foi reduzir o índice de recidivas. A exérese da cápsula como técnica de abordagem cirúrgica é discutível. Se por um lado obtém-se material para exame anatomopatológico, por outro, sua retirada pode de alguma forma lesar o parênquima ovariano normal. Existe a controvérsia sobre a possibilidade de atipias com potencial para malignização em células da cápsula ${ }^{15}$. Por isso toda cápsula deveria ser retirada e não destruída com laser ou corrente elétrica. Esta tese no entanto foi contestada por Donnez et al. ${ }^{5}$. Segundo Brosens et al. ${ }^{3}$, a ovarioscopia guiando a biópsia pode ser suficiente para se obter material adequado para descartar a presença de malignidade.

Este estudo, apesar de casuística pequena, mostra bons resultados, baixo índice de recidiva e boa aceitação pelas pacientes do protocolo utilizado. Todas as recidivas ocorreram em pacientes com endometriomas com diâmetro maior que $3 \mathrm{~cm}$, o que salienta a importância do diagnóstico precoce da patologia. Os trabalhos citados mostram que, em geral, a retirada da cápsula ou sua destruição tem resultados semelhantes. A simples drenagem tem índices elevados de recidiva. A vantagem da cistectomia em relação à cauterização é a possibilidade do estudo anatomopatológico. A afirmação de Donnez et al. ${ }^{6}$ de que a cistectomia é mais lesiva ao ovário do que a vaporização com laser carece de confirmação. Estudos com maior número de pacientes são necessários para se avaliar melhor a eficácia desta forma de tratamento. 


\section{SUMMARY}

Purpose: to evaluate the videolaparoscopic surgical management of 32 patients with ovarian endometrioma.

Method: retrospective study of thirty-two patients admitted to the Gynecologic Endoscopy Section - Gynecology and Obstetrics Department of the Hospital do Servidor Público Estadual "Francisco Morato de Oliveira" - São Paulo Brazil with clinical and ultrasonographic diagnosis of ovarian endometrioma, who have been submitted to videolaparoscopic surgery. Eleven of them had endometriomas less than $3 \mathrm{~cm}$ and had the tumor emptied and the capsule excised at the first laparoscopy. Twentyone patients with endometriomas larger than $3 \mathrm{~cm}$ were submitted to emptying and washing of the cyst at the first laparoscopy. These women used GnRH analogues for four months (1 dose each month) and were then submitted to a second laparoscopy when the capsule was excised. Histopathologic study was performed in the surgical tissues of all cases. The early results of the procedure and the recurrence rate were evaluated.

Results: there were no surgical intercurrent episodes or postsurgical complications. The problem was solved in all but three patients among the 21 with endometriomas larger than $3 \mathrm{~cm}$. These patients presented recurrence of the disease within a period of 6 to 12 months.

Conclusion: the videolaparoscopic surgical management of ovarian endometriomas, with capsule excision, showed good results and small number of recurrences.

KEY WORDS: Endometriosis. Videolaparoscopy. GnRH analogues.

\section{Referências}

1. Donnez J. Endoscopic management of peritoneal and ovarian endometriosis. In: Donnez J, Nisolle M, editors. An Atlas of Laser Operative Laparoscopy and Hysteroscopy. 2nd ed. New York: Parthenon; 1994. p.63.

2. Brosens IA, Puttemans PJ, Deprest J. The endoscopic localization of endometrial implants in the ovarian chocolate cyst. Fertil Steril 1994; 61:1034-8.

3. Brosens IA, Van Ballaer P, Puttemans PJ, Deprest $J$ Reconstruction of the ovary containing large endometriomas by an extraovarian endosurgical technique. Fertil Steril 1996; 66:517-21.
4. Nezhat F, Nezhat C, Allan CJ, Metzger DA, Sears DL. Clinical and histologic classification of endometriomas. Implications for a mechanism of pathogenesis. J Reprod Med 1992; 37:771-6.

5. Donnez J, Nisolle M, Gillet N, Smets M, Bassil S, Casanasas-Roux F. Large ovarian endometriomas. Hum Reprod 1996; 11:641-6.

6. Donnez J, Nisolle M, Gillerot S, Anaf V, ClerckxBraun F, Casanasas-Roux F. Ovarian endometrial cysts: the role of gonadotropin-releasing hormone agonist and/or drainage. Fertil Steril 1994; 62:63-6.

7. Muzii L. The impact of preoperative gonadotropinreleasing hormone agonist treatment on laparoscopic excision of ovarian endometriotic cysts. Fertil Steril 1996; 65:1235-7.

8. Sutton CJ, Ewen SP, Jacobs SA, Whitelaw NL. Laser laparoscopic surgery in the treatment of ovarian endometriomas. J Am Assoc Gynecol Laparosc 1997; 4:319-23.

9. Busacca M, Marana R, Caruana P, et al. Recurrence of ovarian endometrioma after laparoscopic excision. Am J Obstet Gynecol 1999; 180:51923.

10.Saleh A, Tulandi T. Reoperation after laparoscopic treatment of ovarian endometriomas by excision and by fenestration. Fertil Steril 1999; 72:3224.

11.Hemmings R, Bissonnette F, Bouzayen R. Results of laparoscopic treatments of ovarian endometriomas: laparoscopic ovarian fenestration and coagulation. Fertil Steril 1998; 70:527-9.

12.Beretta P, Franchi M, Ghezzi F, Busacca M, Zupi E, Bolis P. Randomized clinical trial of two laparoscopic treatments of endometriomas: cystectomy versus drainage and coagulation. Fertil Steril 1998; 70:1176-80.

13.Canis M, Mage G, Manhes H, Pouly JL, Wattiez A, Bruhat MA. Laparoscopic treatment of endometriosis. Acta Obstet Gynecol Scand Suppl 1989; 150:15-20.

14.Fayez JA, Vogel MF. Comparison of different treatment methods of endometriomas by laparoscopy. Obstet Gynecol 1991; 78:660-5.

15.Czernobilsky B, Morris WJ. A histologic study of ovarian endometriosis with emphasis on hyperplasic and atypical changes. Obstet Gynecol 1979; 53:318-23. 